2 - ORIGINAL ARTICLE

ISCHEMIA-REPERFUSION

\title{
Dexmedetomidine preconditioning inhibits the long term inflammation induced by renal ischemia/reperfusion injury in rats $^{1}$
}

\author{
Guiyong Liu', Hongfei Song ${ }^{\mathrm{II}}$, Lili Qiu ${ }^{\mathrm{III}}$, Anren $\mathrm{He}^{\mathrm{IV}}$, Fangfang Tong ${ }^{\mathrm{V}}$, Qifu Wan ${ }^{\mathrm{IV}}$, Xin Wang ${ }^{\mathrm{IV}}$, Yunfang Xia ${ }^{\mathrm{IV}}$, Lequn Huang ${ }^{\mathrm{IV}}$ \\ DOI: http://dx.doi.org/10.1590/S0102-865020160010000002 \\ IPhD, Department of Urology, Qianjiang central Hospital, Hubei, P.R., China. Design and supervise all phases of the study, analysis of data, manuscript \\ writing. \\ "Master, Department of Urology, Qianjiang central Hospital, Hubei, P.R., China. Acquisition and analysis of data. \\ "IB Bachelor, Department of Endocrinology, Qianjiang central Hospital, Hubei, P.R., China. Acquisition of data, critical revision. \\ ${ }^{\mathrm{IV}}$ Bachelor, Department of Urology, Qianjiang central Hospital, Hubei, P.R., China. Acquisition of data. \\ ${ }^{v}$ Master, Department of Clinical Laboratory, Qianjiang central Hospital, Hubei, P.R., China. Acquisition of data.
}

\begin{abstract}
PURPOSE: To investigate the protective effects of dexmedetomidine (Dex) against renal ischemia/reperfusion injury (IRI).

METHODS: Sprague-Dawley rats were randomly divided to sham group, IRI group and Dex group. The SD rats were subjected to $45 \mathrm{~min}$ of ischemia followed by eight weeks of reperfusion. Prior to ischemia, rats were either treated with Dex or not. Blood samples were collected for the detection of blood urea nitrogen (BUN) and creatinine (Cr) levels. Immunohistochemistry was performed for CD3 T-cell infiltrates. Real-time PCR and western blot were detected for the expression of TNF- $\alpha$, IL-1 $\beta$, ICAM-1, HMGB1 and TLR4. RESULTS: Compared with sham group, renal IRI significantly increased the serum levels of BUN and Cr. The H\&E staining indicated that renal IRI resulted in obvious renal injury and immunohistochemistry found that there were more CD3 T-cell infiltrates in IRI group. Also, renal IRI upregulated the expression of TNF- $\alpha$, IL-1 $\beta$, ICAM-1, HMGB1 and TLR4. However, all these changes were alleviated by the treatment with Dex.
\end{abstract}

CONCLUSIONS: Dexmedetomidine has beneficial effects on long term inflammation induced by renal ischemia/reperfusion injury. Its mechanisms may be achieved through inhibiting the HMGB1/TLR4 pathway to exert protective effects.

Key words: Dexmedetomidine. Ischemia. Reperfusion Injury. Inflammation. Rats. 


\section{Introduction}

Renal ischemia and reperfusion injury (IRI), which usually occurs in patients undergoing kidney transplantation, can increase the rates of acute kidney failure, delayed graft function, and early mortality ${ }^{1}$. In the pathogenesis of renal IRI, there has some factors implicated in it, such as vasoconstriction, oxygenderived free radicals, loss of proximal tubular cell polarity ${ }^{2}$. The acute inflammation caused by renal IRI is characterized by the induction of an inflammatory cytokine cascade, expression of adhesion molecules, and cellular infiltration ${ }^{2}$. Therefore, the strategy to inhibit inflammatory response in IRI will afford effective protection to reduce tissue injury and improve prognosis.

Dexmedetomidine (Dex) is a highly selective $\alpha 2$ adrenoreceptor agonist, with sedative, analgesic, sympatholytic and hemodynamic stabilizing effects ${ }^{3}$. There is accumulating evidence to suggest that Dex has organoprotective effects, including kidney, brain, heart and intestine ${ }^{4-7}$. It has been reported that Dex can increase urine output through the inhibition of vasopressin secretion and the enhancement of renal glomerular filtration. Also, it has been found that Dex can alleviate renal IRI through the effect of cytoprotection ${ }^{8}$. However, the protective mechanism of Dex against renal IRI still remains unclear.

Recently, the effect of HMGB1 and TLR4 has been investigated in organic IRI, including heart, liver and brain ${ }^{9-11}$. High mobility group box 1 (HMGB1) can be released from necrotic cells to the extracellular or secreted by the immune cells after proinflammatory stimulation ${ }^{12}$. As a alarmin, HMGB1 in the extracellular can initiate inflammatory reaction caused by IRI, which has been demonstrated by the model of hepatic IRI in mice ${ }^{13}$. Toll-like receptors are a family of transmembrane proteins. Its activation leads to over-expressions of inflammatory cytokine gene leading to pro-inflammatory response ${ }^{14}$. The previous study was found that Dex could have beneficial effect against intestinal IRI through the reduction of inflammation ${ }^{15}$. However, whether Dex could inhibit inflammation caused by renal IRI still remains unknown. Therefore, the aim of this study was to determine whether Dex could attenuate long term inflammation induced by renal IRI and its possible mechanism.

\section{Methods}

\section{Animal model of $I / R$}

This project was approved by the committee of experimental animals of Wuhan University, and the procedures were carried out according to the routine animal-care guidelines.

All adult male SD rats $(220-250 \mathrm{~g})$ were from the Center of Experimental Animals in Medical College, Wuhan University. Briefly, rats were anesthetized with pentobarbital $(45 \mathrm{mg} / \mathrm{kg})$ and placed on a homeothermic table in order to maintain core body temperature at $37^{\circ} \mathrm{C}$. The midline laparotomy was made and right nephrectomy was performed. After it, the left kidney was subjected to $45 \mathrm{~min}$ of ischemia followed by eight weeks of reperfusion.

All animals were divided into three different group: sham group, IRI group and Dex group. Each group had 6 rats. In sham group, only the right kidneys were removed. In IRI and Dex group, the left kidney vessels were clamped for $45 \mathrm{~min}$ followed by eight weeks of reperfusion. In Dex group, dexmedetomidine $(100 \mu \mathrm{g} /$ $\mathrm{kg}$, i.p., based on the previous study ${ }^{16}$ ) was administrated $30 \mathrm{~min}$ before ischemia. While rats in IRI group and sham group were simultaneously injected the equal volume of PBS. At eight weeks of reperfusion period, the left kidneys were removed for the following experiments and the blood samples were collected for the detection of blood urea nitrogen (BUN) and creatinine $(\mathrm{Cr})$ levels.

\section{Sampling}

The left kidney was removed under fully maintained anesthesia. After removal, the kidney was fixed in $10 \%$ phosphatebuffered formalin or immediately frozen, and stored at $-80^{\circ} \mathrm{C}$ for following experiments.

At eight weeks after IRI in every group, $1 \mathrm{ml}$ blood samples were taken and performed according to directions of the commercial kits (Nanjing Jiancheng Co., China). The absorbance was measured by spectrophotometer and then the concentrations of BUN and Cr were calculated.

Proteinuria was determined at the end of eight weeks from 24-h urine collections according to directions of the commercial kits (Nanjing Jiancheng Co., China).

\section{Histologic examinations}

After the kidney fixed in $10 \%$ phosphate -buffered formalin, it was embedded with paraffin and sectioned at $4-\mu \mathrm{m}$ thick. The sections were deparaffinized and hydrated gradually, and stained with hematoxylin and eosin (H\&E). Morphologic assessments were observed by an experienced renal pathologist who was unaware of the treatments. 


\section{Masson's trichrome staining}

After the kidney fixed in $10 \%$ phosphate -buffered formalin, it was embedded with paraffin and sectioned at $4-\mu \mathrm{m}$ thick. The sections were deparaffinized and hydrated gradually, and stained with Masson's trichrome. Morphologic assessments were observed by an experienced renal pathologist who was unaware of the groups and treatments.

\section{Immunohistochemistry}

The immunohistochemical staining of CD3 was performed using UltraVision ${ }^{\mathrm{TM}}$ Quanto Detection System HRP DAB sample kit (Thermo Scientific). The CD3 antibody (1:100 dilution; SantaCruz Biotechnology, Santa Cruz, CA) was incubated at $4^{\circ} \mathrm{C}$ overnight. The steps were following the manufacturer's instruction.

\section{Realtime PCR}

Total RNA were isolated using Trizol reagent (Invitrogen) and RNA concentration was obtained by spectrophotometer. Single-stranded cDNA was synthesized using the cDNA synthesis kit (Takara, Kyoto, Japan) according to the procedures. Reverse transcription-polymerase chain reaction (PCR) was performed with the Applied Biosystems SYBR Green mix kit (Applied Biosystems, CA, USA). The primers used were as follows: TNF- $\alpha$ forward primer 5'- CTTCTCATTCCTGCTCGTGG -3' , and TNF- $\alpha$ reverse primer 5'- TCCGCTTGGTGGTTTGCTAC - 3' (Gen-Bank accession number NM_012675.3); IL-1 $\beta$ forward primer 5'- ACTATGGCAACTGTCCCTGAAC -3' , and IL-1 $\beta$ reverses primer 5'- GTGCTTGGGTCCTCATCCTG -3' (GenBank accession number NM_031512.2); ICAM-1 forward primer 5'- GGGATGGTGAAGTCTGTCAA -3' , and ICAM-1 reverses primer 5'- GGCGGTAATAGGTGTAAATGG -3' (GenBank accession number NM_012967). $\beta$-actin was used as a housekeeping gene. The data were presented as a ratio of genes to $\beta$-actin mRNA (sense: 5 '- TGCTATGTTGCCCTAGACTTCG $-3^{\prime}$ and antisense: $5^{\prime}-$ GTTGGCATAGAGGTCTTTACGG $-3^{\prime}$ and NM_031144).

\section{Western Blot analysis}

Total proteins were extracted, and quantified using Bicinchoninic acid method. Then, equivalent weights of protein (40 $\mu \mathrm{g} /$ lane) was separated on $10 \%$ SDS-PAGE gels and then transferred to nitrocellulose membrane. The membranes were blocked with 5\% non-fat milk in TBST buffer and then incubated with the following primary antibodies: HMGB1 (1:1000 dilution; Abcam, Cambridge, MA) and TLR4 (1:1000 dilution; SantaCruz Biotechnology, Santa Cruz, CA). Subsequently, after being washed twice with TBST, the membranes were incubated with secondary antibody conjugated with horseradish peroxidase at 1:2000 dilution. Specific bands were visualized by using an enhanced chemiluminescence detection kit.

\section{Statistical analysis}

Data were presented as mean \pm SEM. The means of the different groups were compared using one-way ANOVA 3 Student-Newman-Keuls test. Differences were considered statistically significant when $\mathrm{p}<0.05$.

\section{Results}

\section{Functional parameters}

It was manifest from the results that the levels of BUN and $\mathrm{Cr}$ had no significant difference in three groups. But Dex could decrease the level of proteinuria caused by renal IRI (Figure 1).
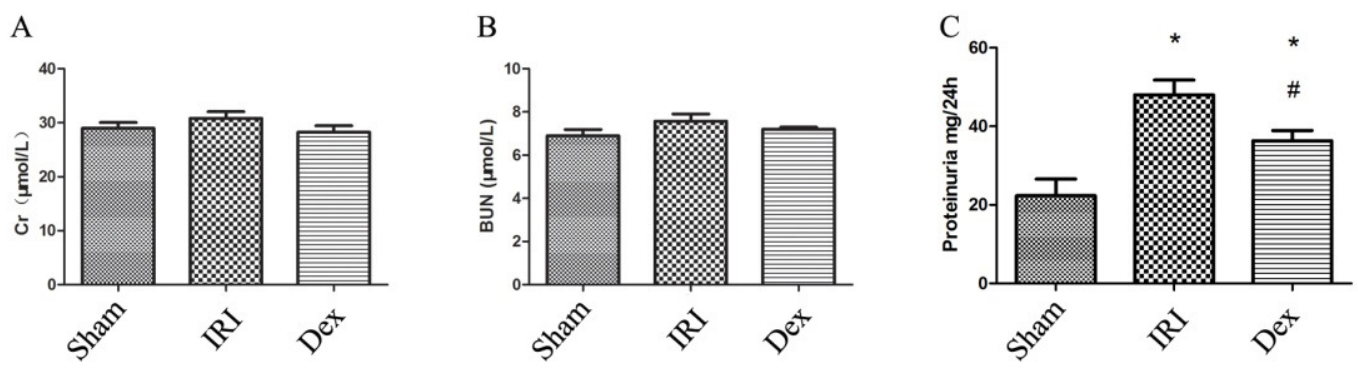

FIGURE 1 - (A) Effects of Dex on the serum Cr concentrations after 45 min of ischemia followed by 8 weeks of reperfusion. (B) Effects of Dex on the serum BUN concentrations after 45 min of ischemia followed by 8 weeks of reperfusion. (C) Effects of Dex on the proteinuria after 45 min of ischemia followed by 8 weeks of reperfusion. Bars represent means \pm SEM $(n=4)$. 


\section{Histopathology}

Compared with sham operated group, renal IRI resulted in significant renal injury, as evidenced by inflammatory infiltration and loss of renal parenchyma characterized by tubular atrophy, rarefaction of peritubular capillaries, and podocyte depletion. However, the treatment with Dex could reduce these severe renal damages (Figure 2).
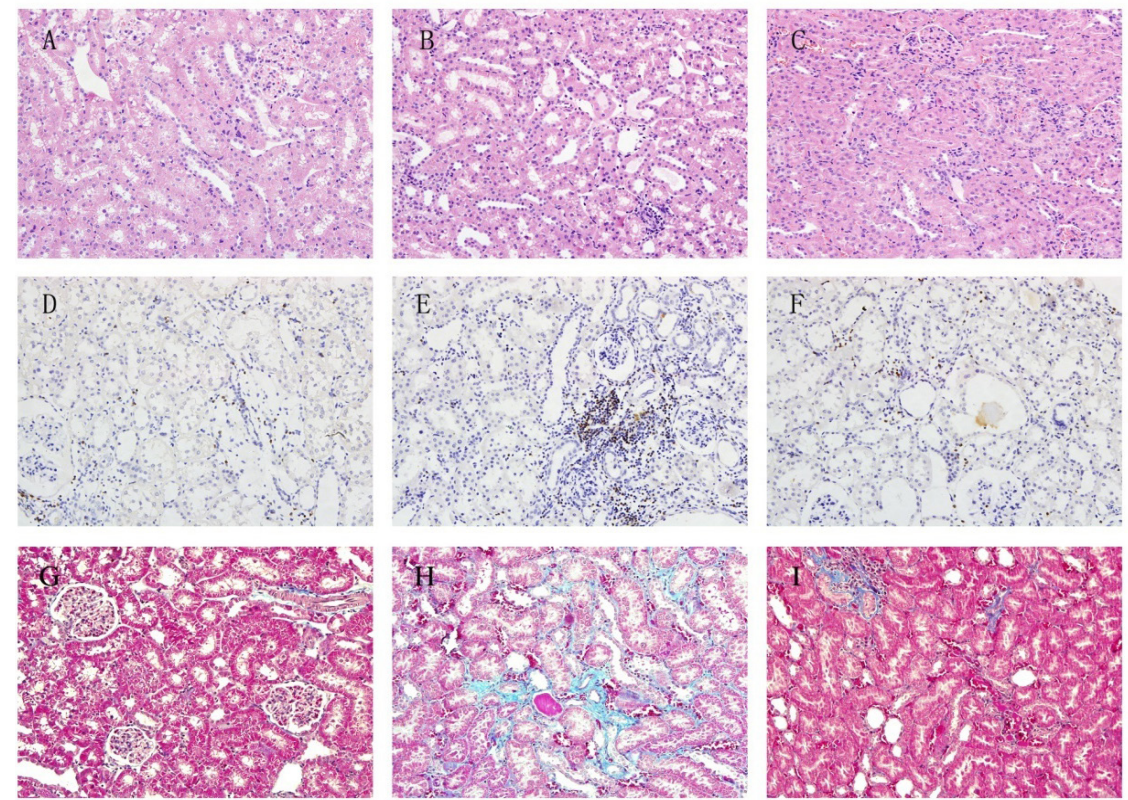

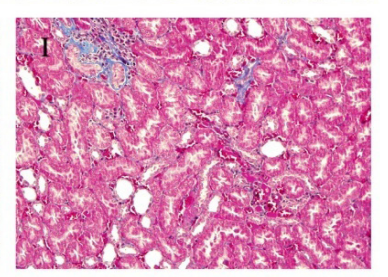

FIGURE 2 - Histologic features were evaluated by H\&E, immunohistochemical and Masson staining. (A, B, C) Representative kidney sections stained with H\&E. (D, E, F) The expression spots of CD3 in kidneys at the end of reperfusion 8 weeks. $(\mathbf{G}, \mathbf{H}, \mathbf{I})$ The fibrosis caused by ischemic damage in kidneys at the end of reperfusion 8 weeks. Sham group $(\mathbf{A}, \mathbf{D}, \mathbf{G})$ : Section from sham-operated rat. IRI $(\mathbf{B}, \mathbf{E}, \mathbf{H})$ : Section from rat subjected to IRI . $\operatorname{Dex}(\mathbf{C}, \mathbf{F}, \mathbf{I})$ : Section from rat treated to Dex. H\&E staining, original magnification $\times 200$; Immunohistochemical staining, original magnification $\times 200$; Masson staining, original magnification $\times 200$.

\section{Immunohistochemistry}

In our study, the expression of CD3 (Figure 2) was detected by immunohistochemical staining. The results revealed that CD3 positive cells were rarely found in sham group. But in IRI group, there were more CD3 T-cell infiltrates. Compared with IRI group, this change was ameliorated in Dex group.

\section{Masson's trichrome staining}

The result revealed that IRI could lead to renal fibrosis and rats treated with Dex had less fibrosis compared with rats in IRI group (Figure 2).

\section{Realtime PCR analysis}

The relative mRNA expression of TNF- $\alpha$, IL- $1 \beta$ and ICAM-1 to $\beta$-actin were shown (Figure 3 ). These mRNA levels were significantly greater in IRI group than those in sham group. However, the treatment with Dex could significantly reduce the mRNA expression of TNF- $\alpha$, IL-1 $\beta$ and ICAM-1 after IRI.
A

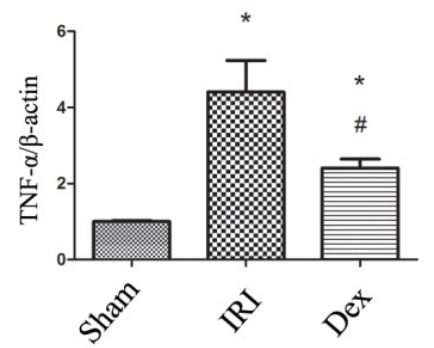

B

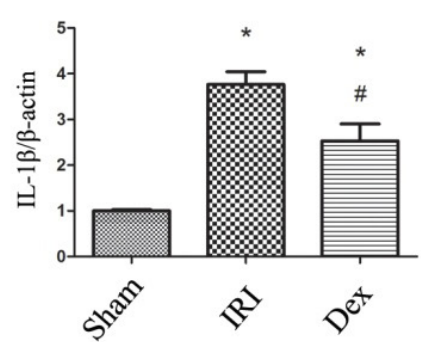

C

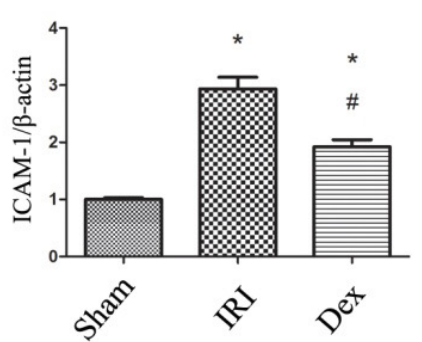

FIGURE 3 - The mRNA level of TNF- $\alpha$, IL-1 $\beta$ and ICAM-1 in the kidney. (A) Effects of Dex on the mRNA level of TNF- $\alpha$ after 45 min of ischemia followed by 8 weeks of reperfusion. (B) Effects of Dex on the mRNA level of IL-1 $\beta$ after 45 min of ischemia followed by 8 weeks of reperfusion. (C) Effects of Dex on the mRNA level of ICAM-1 after 45 min of ischemia followed by 8 weeks of reperfusion. mRNA was standardized for $\beta$-actin mRNA. Bars represent means $\pm \operatorname{SEM}(\mathrm{n}=3) ;{ }^{*} \mathrm{p}<0.05$ versus Sham, $\# \mathrm{p}<0.05$ versus IRI. 


\section{Western Blot analysis}

To investigate the different levels of protein expression, we measured HMGB1 and TLR4 by Western blot (Figure 4).It was obvious from the results that the expressions of HMGB1 and TLR4 were increased after subjected to IRI when compared with sham group. But Dex could attenuate these expression induced by renal IRI.
A

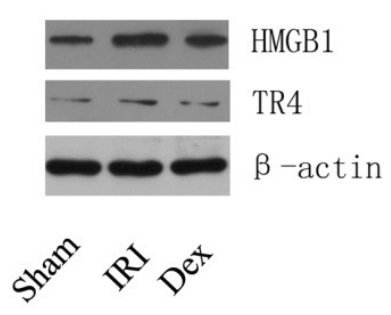

B

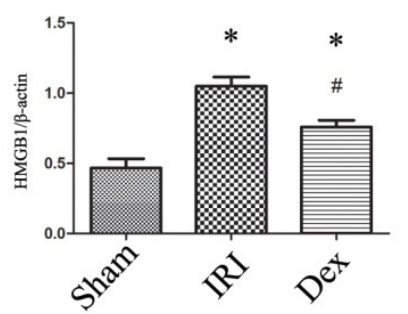

C

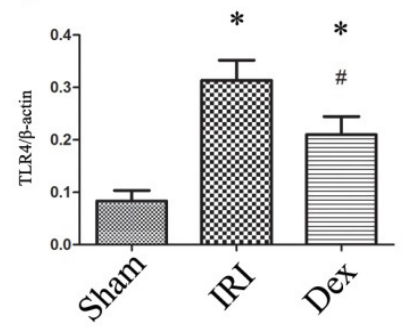

FIGURE 4 - Representative Western blots showed the effects of Dex on HMGB1 and TLR4 expression in the kidney after 45 min of ischemia followed by 8 weeks of reperfusion. $\beta$-actin was used to show equal amounts of protein loading in each lane. (A) Representative Western blots showing the effects of Dex on HMGB1 and TLR4 expression. (B) Relative band densities of HMGB1 to the mean value of the control. (C) Relative band densities of TLR4 to the mean value of the control. Bars represent means $\pm \operatorname{SEM}(\mathrm{n}=3) ;{ }^{*} \mathrm{p}<0.05$ versus Sham, $\# \mathrm{p}<0.05$ versus IRI.

\section{Discussion}

IRI has elicited an increasing interest of the researchers in its impact on such organs as kidney, liver and heart in recent years. Inflammation represents key factor in the occurrence and development of ischemic damage, which is thought to occur secondary to an intense inflammatory response initiated by the infiltration of leukocytes and the production of proinflammatory cytokines after IRI ${ }^{17}$. Previous studies has demonstrated that renal ischemic damage can lead to severe inflammation after 24 hours of reperfusion ${ }^{18}$. In addition, recent study also found that after 12 weeks of reperfusion, renal IRI could result in long term inflammation ${ }^{19}$.

In this study, a 45-min rat renal ischemia model was established to investigate the protective effect of Dex against renal IRI, which was induced by unilateral clamping of renal vascular structures. Renal injury was examined by the renal function and H\&E staining after 8 weeks of reperfusion. At current study, the levels of BUN and $\mathrm{Cr}$ had no significant difference in three groups. But histopathology result showed that renal IRI resulted in severe renal injury, as evidenced by inflammatory infiltration and loss of renal parenchyma characterized by tubular atrophy, rarefaction of peritubular capillaries, and podocyte depletion ${ }^{20}$. However, Dex can significantly reduce these severe renal damage. These results were in accordance with prior study which has shown that although renal function at the end of 12 weeks showed no significant differences among all groups, their results showed that IRI induced severe renal injury ${ }^{19}$.
Dexmedetomidine (Dex) is a highly selective $\alpha 2$ adrenoreceptor agonist, with sedative, analgesic, sympatholytic and hemodynamic stabilizing effects ${ }^{3}$. In addition, it has been reported that Dex has organoprotective effects against ischemic damage, including celebral, hepatic, cardiac, renal and testicular IRI $I^{4,6,7,21,22}$. Gonullu et al. ${ }^{16}$ showed that the preconditioning or postconditioning with the administration of Dex could both reduce the renal IRI histomorphologically, as the renal histopathological score in the IRI group was significantly higher than the groups with Dex. Other studies also demonstrated that the administration with Dex could reduce the number of apoptotic tubular epithelial cells and attenuate renal tissue necrosis and histological lesions in a rat IRI model ${ }^{7,8}$. Besides, wang et al..$^{23}$ showed that innormoglycemic condition Dex could attenuate renal damage caused by IRI, but in hyperglycemic condition there was no difference between Dex and IRI group. These studies just investigate the protective effect of Dex after 24 hours of reperfusion under ischemic stimuli and our data showed that the model of 45 minutes' ischemia and 8 weeks' reperfusion could result in long term tissue damage, including inflammation, as the evidence of $\mathrm{H} \& \mathrm{E}$ staining and immunohistochemical staining with CD3. However, the treatment with Dex prior to ischemia could significantly reduce renal damages caused by IRI and attenuate the CD3 T-cell infiltrates.

A growing body of evidence indicates that the inflammatory response, associated with pro-inflammatory cytokines IL- $1 \beta$, TNF- $\alpha$ and chemotactic cytokine MCP-1, plays a key role in renal dysfunction following IRI ${ }^{24}$. ICAM, as an adhesion molecule, could facilitate leukocytes infiltration and adhesion to 
aggravate the injuries caused by IRI. Si et al. ${ }^{25}$ showed that the treatment with Dex can protect kidney against IRI at 48 hours of reperfusion, accompanied with the downregulation of ICAM-1 and MCP-1 expression. In line with previous study, our data indicated that Dex can significantly decrease the expression of TNF- $\alpha$, IL$1 \beta$ and ICAM- 1 after IRI. This significantly demonstrated Dex has the anti-inflammation effect against the renal IRI.

HMGB-1 is a potent damage-associated molecular patterns released from dying cells during tissue ischemia. It binds to TLR-4 initiating down-stream NF- $\mathrm{B}$ signaling cascade ${ }^{26}$ substantially augmenting the synthesis of pro-inflammatory cytokines such as TNF-a and IL-1 $\beta^{27}$. Recent work by Wu et al. ${ }^{14}$ demonstrated that TLR4-deficient mice were protected from both kidney dysfunction and histological damage induced by renal IRI. Gu et al. ${ }^{7}$ work found that after 24 hours of reperfusion, Dex reduced HMGB1 release and inhibits TLR4 signaling to provide reno-protection. Our data indicated that after 8 weeks of reperfusion, Dex can significantly decrease the expression of HMGB1 and TLR4. This significantly demonstrated Dex could afford long term protection against renal IRI.

Some aspects of the present study deserve further discussion. We used only eight weeks of reperfusion to investigate the effect of Dex on renal ischemic damage. Whether after longer reperfusion time, such as 12 and 16 weeks of reperfusion, Dex also has obvious protective effect against renal IRI may need to be elucidated in further research.

\section{Conclusion}

Dexmedetomidine has the long term protective effect against renal IRI through the alleviation of inflammation.

\section{References}

1. Kosieradzki M, Rowinski W. Ischemia/reperfusion injury in kidney transplantation: mechanisms and prevention. Transplant Proc. 2008;40(10):3279-88. doi: 10.1016/j.transproceed.2008.10.004.

2. Bonventre JV, Weinberg JM. Recent advances in the pathophysiology of ischemic acute renal failure. J Am Soc Nephrol. 2003;14(8):2199210. PMID: 12874476.

3. Farag E, Argalious M, Abd-Elsayed A, Ebrahim Z, Doyle DJ. The use of dexmedetomidine in anesthesia and intensive care: a review. Curr Pharm Des. 2012;18(38):6257-65. PMID: 22762468.

4. Engelhard K, Werner C, Eberspacher E, Bachl M, Blobner M, Hildt E, Hutzler P, Kochs E. The effect of the alpha 2-agonist dexmedetomidine and the N-methyl-D-aspartate antagonist $\mathrm{S}(+)$ ketamine on the expression of apoptosis-regulating proteins after incomplete cerebral ischemia and reperfusion in rats. Anesth Analg. 2003;96(2):524-31.PMID: 12538207.

5. Zhang XY, Liu ZM, Wen SH, Li YS, Li Y, Yao X, Huang WQ, Liu KX. Dexmedetomidine administration before, but not after, ischemia attenuates intestinal injury induced by intestinal ischemiareperfusion in rats. Anesthesiology. 2012;116(5):1035-46. doi: 10.1097/ALN.0b013e3182503964.

6. Yoshitomi O, Cho S, Hara T, Shibata I, Maekawa T, Ureshino H, Sumikawa K. Direct protective effects of dexmedetomidine against myocardial ischemia-reperfusion injury in anesthetized pigs. Shock. 2012;38(1):92-7. doi: 10.1097/SHK.0b013e318254d3fb.

7. Gu J, Sun P, Zhao H, Watts HR, Sanders RD, Terrando N, Xia P, Maze M, Ma D. Dexmedetomidine provides renoprotection against ischemia-reperfusion injury in mice. Crit Care. 2011;15(3):R153. doi: 10.1186/cc10283.

8. Billings FT, Chen SW, Kim M, Park SW, Song JH, Wang S, Herman J, D'Agati V, Lee HT. alpha2-Adrenergic agonists protect against radiocontrast-induced nephropathy in mice. Am J Physiol Renal Physiol. 2008;295(3):F741-8. doi: 10.1152/ajprenal.90244.2008.

9. Zhang T, Yang S, Du J. Protective effects of berberine on isoproterenol-induced acute myocardial ischemia in rats through regulating HMGB1-TLR4 axis. Evid Based Complement Alternat Med. 2014;2014:849783. doi: 10.1155/2014/849783.

10. Mcdonald KA, Huang H, Tohme S, Loughran P, Ferrero K, Billiar T, Tsung A. Toll-like receptor 4 (TLR4) antagonist eritoran tetrasodium attenuates liver ischemia and reperfusion injury through inhibition of high-mobility group box protein B1 (HMGB1) signaling. Mol Med. 2014;20:639-48. doi: 10.2119/molmed.2014.00076.

11. Zhang J, Wu Y, Weng Z, Zhou T, Feng T, Lin Y. Glycyrrhizin protects brain against ischemia-reperfusion injury in mice through HMGB1TLR4-IL-17A signaling pathway. Brain Res. 2014;1582:176-86. doi: 10.1016/j.brainres.2014.07.002.

12. Tang D, Shi Y, Kang R, Li T, Xiao W, Wang H, Xiao X. Hydrogen peroxide stimulates macrophages and monocytes to actively release HMGB1. J Leukoc Biol. 2007;81(3):741-7. doi: 10.1189/ jlb.0806540.

13. Tsung A, Sahai R, Tanaka H, Nakao A, Fink MP, Lotze MT, Yang H, Li J, Tracey KJ, Geller DA, Billiar TR. The nuclear factor HMGB1 mediates hepatic injury after murine liver ischemia-reperfusion. J Exp Med. 2005;201(7):1135-43. doi: 10.1084/jem.20042614.

14. Wu H, Chen G, Wyburn KR, Yin J, Bertolino P, Eris JM, Alexander SI, Sharland AF, Chadban SJ. TLR4 activation mediates kidney ischemia/reperfusion injury. J Clin Invest. 2007;117(10):2847-59. doi: 10.1172/JCI31008.

15. Sun Y, Gao Q, Wu N, Li SD, Yao JX, Fan WJ. Protective effects of dexmedetomidine on intestinal ischemia-reperfusion injury. Exp Ther Med. 2015;10(2):647-52. doi: 10.3892/etm.2015.2561.

16. Gonullu E, Ozkardesler S, Kume T, Duru LS, Akan M, Guneli ME, Ergur BU, Meseri R, Dora O. Comparison of the effects of dexmedetomidine administered at two different times on renal ischemia/reperfusion injury in rats. Braz $\mathrm{J}$ Anesthesiol. 2014;64(3):152-8. doi: 10.1016/j.bjane.2013.06.002.

17. $\mathrm{Du} \mathrm{X}, \mathrm{Hu} \mathrm{X}$, Wei J. Anti-inflammatory effect of exendin-4 postconditioning during myocardial ischemia and reperfusion. $\mathrm{Mol}$ Biol Rep. 2014;41(6):3853-7. doi: 10.1007/s11033-014-3252-0.

18. Wang L, Liu XH, Chen H, Chen ZY, Weng XD, Qiu T, Liu L. Picroside II protects rat kidney against ischemia/reperfusioninduced oxidative stress and inflammation by the TLR4/NFkappaB pathway. Exp Ther Med. 2015;9(4):1253-8. doi: 10.3892/ etm.2015.2225.

19. Weng X, Shen H, Kuang Y, Liu X, Chen Z, Zhu H, Jiang B, Zhu G, Chen $\mathrm{H}$. Ischemic postconditioning inhibits the renal fibrosis induced by ischemia-reperfusion injury in rats. Urology. 2012;80(2):481-4. doi: 10.1016/j.urology.2012.02.054.

20. Liu Y. Renal fibrosis: new insights into the pathogenesis and therapeutics. Kidney Int. 2006;69(2):213-7. doi: 10.1038/ sj.ki.5000054. 
Liu G et al.

21. Sahin T, Begec Z, Toprak HI, Polat A, Vardi N, Yucel A, Durmus M, Ersoy MO. The effects of dexmedetomidine on liver ischemiareperfusion injury in rats. J Surg Res. 2013;183(1):385-90. doi: 10.1016/j.jss.2012.11.034.

22. Tuglu D, Yuvanc E, Yilmaz E, Gencay IY, Atasoy P, Kisa U, Batislam E. The antioxidant effect of dexmedetomidine on testicular ischemia-reperfusion injury. Acta Cir Bras. 2015;30(6):414-21. doi: 10.1590/S0102-865020150060000007.

23. Wang H, Chen H, Wang L, Liu L, Wang M, Liu X. Acute hyperglycemia prevents dexmedetomidine-induced preconditioning against renal ischemia-reperfusion injury. Acta Cir Bras. 2014;29(12):812-8. doi: 10.1590/S0102-86502014001900008.

24. Voss A, Bode G, Kerkhoff C. Double-stranded RNA induces IL-8 and MCP-1 gene expression via TLR3 in HaCaT-keratinocytes. Inflamm Allergy Drug Targets. 2012;11(5):397-405. PMID: 22762377.

25. Si Y, Bao H, Han L, Shi H, Zhang Y, Xu L, Liu C, Wang J, Yang X, Vohra A, Ma D. Dexmedetomidine protects against renal ischemia and reperfusion injury by inhibiting the JAK/STAT signaling activation. J Transl Med. 2013;11:141. doi: 10.1186/1479-5876-11141.

26. O'Neill LA, Bowie AG. The family of five: TIR-domain-containing adaptors in Toll-like receptor signalling. Nat Rev Immunol. 2007;7(5):353-64. doi: 10.1038/nri2079.

27. Akira S, Takeda K, Kaisho T. Toll-like receptors: critical proteins linking innate and acquired immunity. Nat Immunol. 2001;2(8):67580. doi: $10.1038 / 90609$.

\section{Correspondence:}

Dr. Guiyong Liu

Department of Urology

Qianjiang central Hospital, No. 22

Zhanghuazhong Road, Qianjiang 433100

Hubei Province, P.R. China

Phone: (86)0728-6298147

drliugy@126.com.

Received: Set 15, 2015

Review: Nov 18, 2015

Accepted: Dec 13, 2015

Conflict of interest: none

Financial source: none

${ }^{1}$ Research performed at Department of Urology, Qianjiang central Hospital, Hubei, P.R., China. 